\title{
Spatio-Temporal Beam Dynamics in Multimode Nonlinear Optical Fibers
}

\author{
K. Krupa ${ }^{1-3}$, D. Modotto ${ }^{1}$, V. Couderc ${ }^{2}$, A. Barthelemy ${ }^{2}$, A. Tonello ${ }^{2}$, G. Millot ${ }^{3}$, and S. Wabnitz ${ }^{1,4}$ \\ ${ }^{1}$ Dipartimento di Ingegneria dell'Informazione, Università di Brescia, via Branze 38, 25123 Brescia, Italy \\ ${ }^{2}$ Université de Limoges, XLIM, UMR CNRS 7252, 123 Avenue A. Thomas, 87060 Limoges, France \\ ${ }^{3}$ Université Bourgogne Franche-Comté, ICB UMR CNRS 6303, 9 Avenue A. Savary, 21078 Dijon, France \\ ${ }^{4}$ Novosibirsk State University, 1 Pirogova str., Novosibirsk 630090, Russia \\ Tel: (39)030-3715846,Fax: (39)030-380014,e-mail: stefan.wabnitz@unibs.it
}

\begin{abstract}
We overview recent advances in the spatio-temporal nonlinear dynamics of optical pulses propagating in multimode optical fibers. The Kerr effect leads to spatial beam self-cleaning in a graded-index multimode optical fiber, followed by sideband series generation spanning multiple octaves. Effectively single mode supercontinuum spanning from the visible to the mid-infrared was also demonstrated. Enhancement of Kerr beam self-cleaning was observed in active fiber with quasi-step index profile. Moreover, mutual self-cleaning was recently reported for both the fundamental and the second harmonic beams in optically poled multimode fibers with cubic and quadratic nonlinearity.
\end{abstract}

Keywords: optical fibers, nonlinear optics, optical amplifiers, second-harmonic generation.

\section{INTRODUCTION}

Applications of multimode optical fibers (MMFs) have been quite limited so far, because of their inherent inability to maintain a good beam quality owing to modal scrambling. Nevertheless, in recent years the use of MMFs for increasing the transmission capacity of fiber links and the output power of fiber lasers has been extensively investigated. Nonlinear MMFs exhibit complex spatio-temporal propagation dynamics [1]. In the anomalous dispersion regime, femtosecond multimode optical solitons were observed in graded-index (GRIN) MMFs [2], and exploited for controlled generation of ultra-wideband dispersive wave series [3,4].

\section{THEORY}

The spatio-temporal evolution of the complex envelope $A(x, y, z, t)$ of a light wave in a MMF may be described in terms of the generalized 3-D nonlinear Schrödinger equation (GNLSE3D) [5]

$$
\frac{\partial A}{\partial z}-i \frac{1}{2 k_{0}} \nabla_{\perp}^{2} A+i \frac{\kappa^{\prime \prime}}{2} \frac{\partial^{2} A}{\partial t^{2}}+i \frac{k_{0} \Delta}{\rho^{2}} r^{2} A=i k_{0} \frac{n_{2}}{n_{c o}}\left(1-f_{R}\right)|A|^{2} A+i k_{0} \frac{n_{2}}{n_{c o}} f_{R} A \int h(\tau)|A(t-\tau)|^{2} d \tau
$$

where $k_{0}=\omega_{0} n_{c o} / c, \Delta=\left(n_{c o}^{2}-n_{c l}^{2}\right) / 2 n_{c o}^{2}, \omega_{0}$ is the carrier angular frequency of the pump laser, $n_{c o}$ and $n_{c l}$ are the fiber core and cladding refractive indexes, $r$ is the fiber core radius, $h(t)$ is the Raman temporal response of the fiber, and $f_{R}=0.18$. The two terms on the right-hand side of eq.(1) represent the instantaneous and the delayed Raman contributions to fiber nonlinearity, respectively.

\section{KERR BEAM SELF-CLEANING}

For beam powers above a certain threshold, the Kerr effect leads to a strong reduction of modal noise in MMFs.
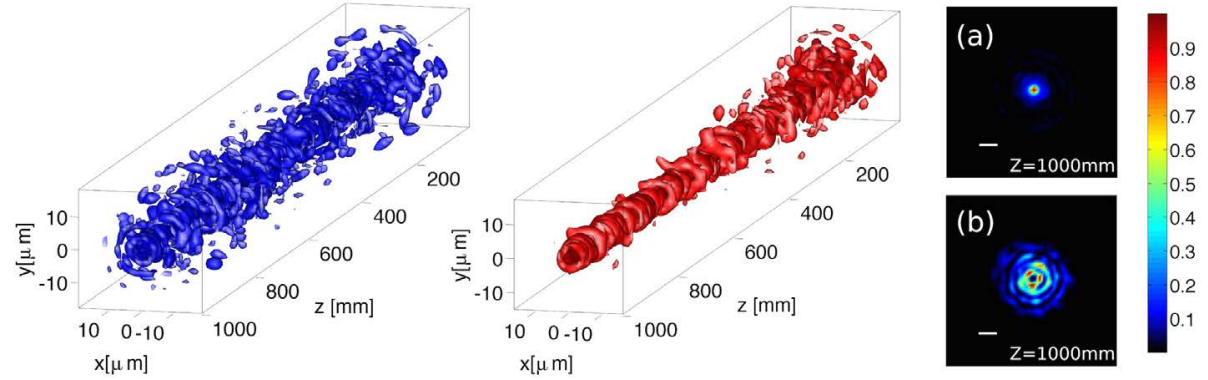

Figure 1. Simulations showing beam self-cleaning in lossless GRIN MMF. Left: speckled beam evolution at $0.5 \mathrm{MW} / \mathrm{cm}^{2}$ (low power); Middle: self-cleaning at $5 \mathrm{GW} / \mathrm{cm}^{2}$ (high power); Right: output beam at $5 \mathrm{GW} / \mathrm{cm}^{2}$ (a) and $0.5 \mathrm{MW} / \mathrm{cm}^{2}$ (b) [5]. 


\subsection{Conservative Self-Cleaning}

Spatial beam self-cleaning induced by the Kerr effect was first theoretically and experimentally investigated in the virtually lossless (or conservative) propagation regime, by using short $(1-10 \mathrm{~m})$ spans of GRIN MMF and quasi-CW sub-nanosecond pulses from an amplified Nd:YAG microchip laser at $1064 \mathrm{~nm}$ [5]. Figure 1 illustrates the numerical demonstration, by solving the eq. (1), of Kerr self-cleaning towards a well-defined, and robust bell-shaped transverse profile after propagation of just over $1 \mathrm{~m}$ [5]. Here mode coupling was introduced by adding a random input spatial phase distribution. In the experiments shown in the left panel of Fig. 2, we confirmed a robust self-cleaning effect to occur for input beam powers around $68 \mathrm{~kW}$. By the cut-back method, Fig. 2(b-f) clearly show the spatial evolution towards the fundamental fiber mode, sitting on a low-power background. Moreover, Fig. 2(a, b) show that beam scrambling in the initial $20 \mathrm{~cm}$ of MMF always occurs, both in the linear and in the nonlinear regime. The presence of Kerr-induced spatial beam cleaning was experimentally shown by Liu et al. to extend down to the 80 fs pump pulse regime [6].
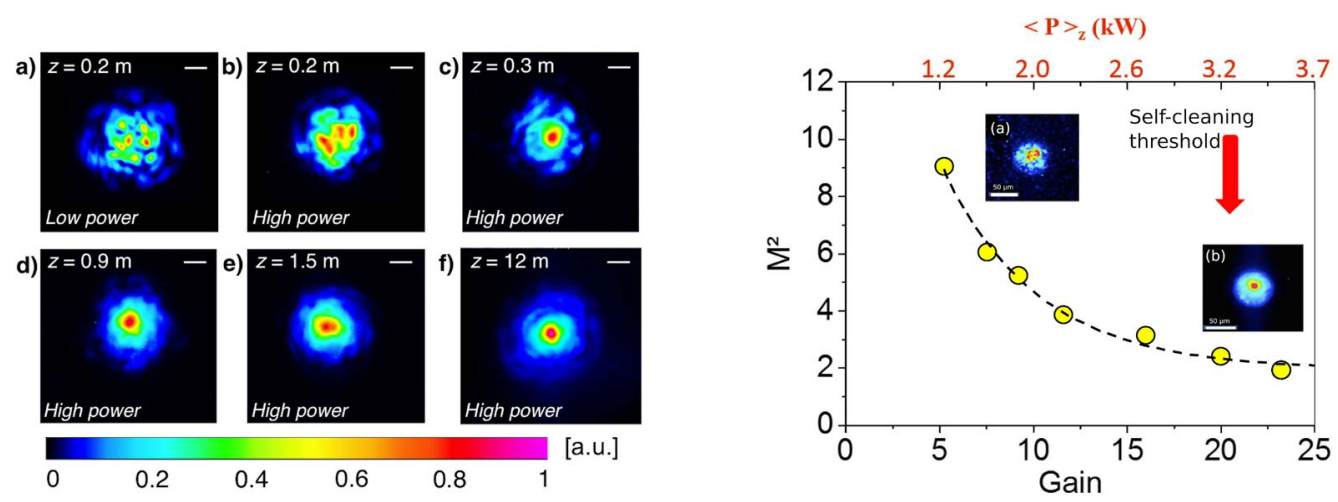

Figure 2. Experiments of Kerr-beam self-cleaning in (left) lossless GRIN MMF (high input power $=68 \mathrm{~kW}$, low input power $=0.007 \mathrm{~kW}, z$-fiber length) [5]; (right) pumped Ytterbium-doped MMF (input signal power $=0.5 \mathrm{~kW}$ and fiber length $=3 \mathrm{~m}$ ) [7]; $\langle P\rangle_{z}$ is a path average power.

\subsection{Dissipative Self-Cleaning}

Next, we experimentally investigated the role of loss and gain in Kerr-induced beam self-cleaning in a $3 \mathrm{~m}$ - long step-index Ytterbium doped MMF (Y-MMF). We observed that dissipative effects surprisingly lead to an increased efficiency of the self-cleaning process: in fact, in the lossless case it is challenging to obtain selfcleaning in a step-index MMF. In particular, using a pumped active MMF permits to reduce the input beam power for self-cleaning to about $0.5 \mathrm{~kW}$ (from $40 \mathrm{~kW}$ measured in the passive regime of Y-MMF) [7]. Note that this value is significantly lower than the $7 \mathrm{~kW}$ threshold in the lossless case of a GRIN fiber of similar length. The right panel of Fig. 2 illustrates the substantial improvement of the output beam quality as the self-cleaning threshold is reached, corresponding to a power gain $G=20$.

\section{SPATIO-TEMPORAL INSTABILITIES}

\subsection{Sideband Series}

Using an intense laser beam with wavelength in the normal dispersion of a GRIN MMF, and with picosecond to nanosecond pump pulses, sideband series spanning from the visible till the mid-infrared (MIR) could be generated $[8,9]$. The main mechanism for sideband generation is the parametric instability of quasi-CW pulses, that is self-induced by the intensity oscillations resulting from the periodic beam self-imaging effect [10]: hence it was termed geometric parametric instability (GPI) [8]. Subsequently, GPI interacts with Raman scattering on the long-wavelength side of the input beam [9].

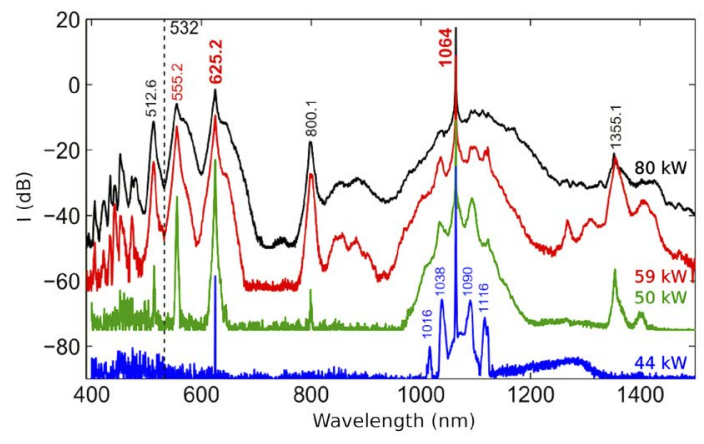

Figure 3. Experimental spectra from a few mode $1 \mathrm{~m}$ - long GRIN fiber, for different pump peak powers [11]. 
Interestingly, as shown in Fig. 3, far-detuned parametric frequency conversion may also occur in a few mode GRIN fiber. Here multiple sidebands spanning in the visible down to $405 \mathrm{~nm}$ and in the near infrared up to $1953 \mathrm{~nm}$ are generated by means of cascaded inter-modal four-wave mixing processes [11].

\subsection{Supercontinuum Generation}

As the peak power of the beam pulses in a GRIN MMF is increased above the thresholds for self-cleaning and GPI sideband formation, the subsequent interplay between GPI and Raman scattering in GRIN MMFs leads to supercontinuum (SC) generation $[12,13]$. As shown in Fig. 4, the generated SC is not only spectrally flat, but most interestingly it is essentially spatially single-mode, and it has been demonstrated to extend from the visible till the MIR [13]. A time resolved analysis of different spectral regions of the SC has revealed that a significant (up to tenfold) temporal compression may occur in the GPI sidebands [13].
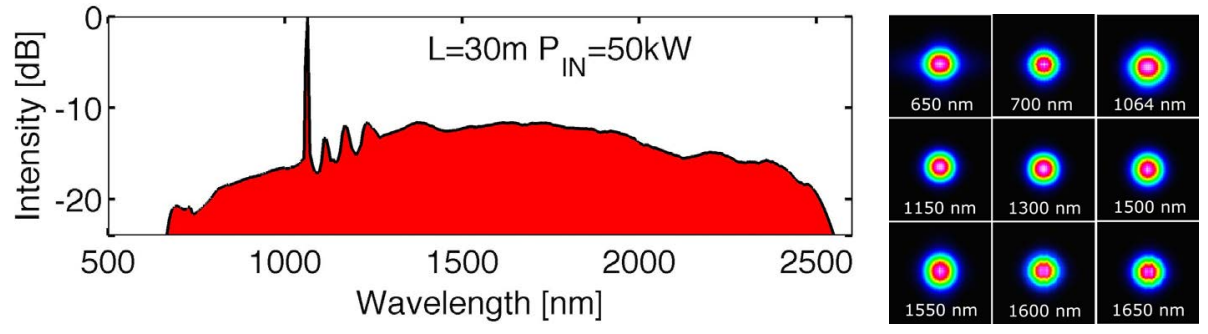

Figure 4. SC spectral intensity from $30 \mathrm{~m}$ - long GRIN MMF (left) and its bell-shaped beam profile (right) [13].

\subsection{Second HarmonicGeneration}

By optically poling a GRIN MMF, a photo-induced charge distribution is generated, which induces a permanent modulated quadratic nonlinearity that is automatically quasi-phase-matched (QPM) for second-harmonic ( $\mathrm{SH}$ ) generation. As shown in the left panel of Fig. 5, we observed that the self-imaging of the pump wave, which is responsible for GPI sidebands around the fundamental frequency (FF), also leads to the generation of a spectral sideband series around the SH [14]. In fact, the periodic FF intensity oscillations lead to the writing of quadratic nonlinearity with multiple periodicities, leading to additional QPM conditions. The Kerr-induced spatial selfcleaning of the FF was accompanied by simultaneous spatial cleaning of the SH beam, as well (see Fig. 5, right panel) [14].
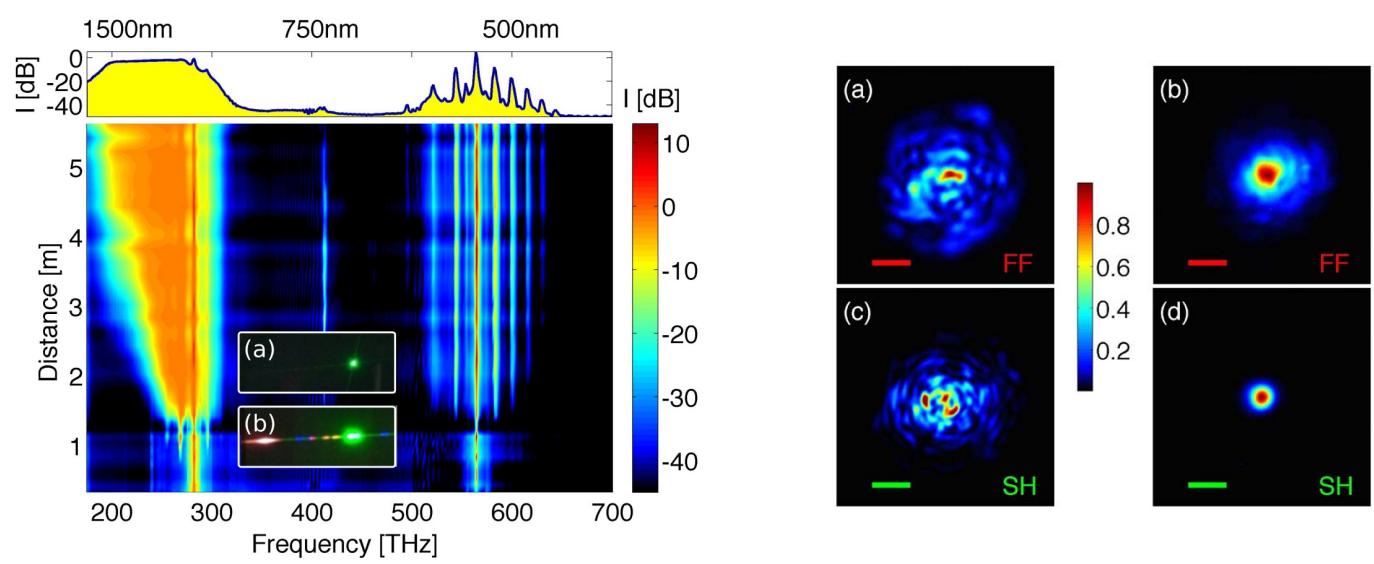

Figure 5. Left: measured spectra from poled GRIN MMF; insets show a photo of the output beam (a) without and (b) with GPI; Right: FF and SH beams from a 2-m long MMF at low (0.94 kW) (a,c) and high (39 kW) (b,d) FF power [14].

\section{CONCLUSIONS}

A series of recent experiments has shown that the nonlinear response of highly multimode fibers may be exploited for achieving the light controlled shaping of the spatial beam at their output. This remarkable finding permits to effectively counter the natural tendency of multimode fibers to scramble the spatial degrees of freedom of light, which has the potential for enabling a wide range of novel applications. We have demonstrated the generation of spatially quasi-single mode octave spanning supercontinuum from a GRIN MMF, and effective second harmonic generation after optical poling. Spatial beam self-cleaning is enhanced in active fiber amplifiers, thus it is expected to provide a key building block for a new class of more efficient high power fiber devices and laser sources. 


\section{ACKNOWLEDGEMENTS}

We gratefully acknowledge support from the iXcore Research Foundation, ANR Labex Action, Ministero dell'Istruzione, dell'Università e della Ricerca (MIUR) (PRIN 2015KEZNYM); Ministry of Education and Science of the Russian Federation (14.Y26.31.0017); Horiba Medical and BPI France (dat@diag project).

\section{REFERENCES}

[1] A. Picozzi, G. Millot, and S. Wabnitz: Nonlinear virtues of multimode fibre, Nat. Photon., vol. 9 , |pp. 289-291, Apr. 2015.

[2] W. H. Renninger and F. W. Wise: Optical solitons in graded-index multimode fibres," Nat. Commun., vol. 4, 1719, Apr. 2013.

[3] L. G. Wright, D. N. Christodoulides, and F. W. Wise: Controllable spatiotemporal nonlinear effects in multimode fibres, Nat. Photon., vol. 9, pp. 306-310, Apr. 2015.

[4] L. G. Wright, S. Wabnitz, D. N. Christodoulides, and F. W. Wise: Ultrabroadband dispersive radiation by spatiotemporal oscillation of multimode waves, Phys. Rev. Lett., vol. 115, 223902, Nov. 2015.

[5] K. Krupa, A. Tonello, B. M. Shalaby, M. Fabert, A. Barthélémy, G. Millot, S. Wabnitz, and V. Couderc: Spatial beam self-cleaning in multimode fiber, Nat. Photon., vol. 11, pp. 237-241, Mar. 2017.

[6] Z. Liu, L. G. Wright, D. N. Christodoulides, and F. W. Wise: Kerr self-cleaning of femtosecond-pulsed beams in graded-index multimode fiber, Optics Letters, vol. 41, pp. 3675-3678, Aug. 2017

[7] R. Guenard, K. Krupa, R. Dupiol, M. Fabert, A. Bendahmane, V. Kermene, A. Desfarges-Berthelemot, J. L. Auguste, A. Tonello, A. Barthélémy, G. Millot, S. Wabnitz, and V. Couderc: Kerr self-cleaning of pulsed beam in an ytterbium doped multimode fiber, Optics Express, vol. 25, pp. 4783-4792, Mar. 2017.

[8] K. Krupa, A. Tonello, A. Barthélémy, V. Couderc, B. Mohamed Shalaby, A. Bendahmane, G. Millot, and S. Wabnitz: Observation of geometric parametric instability induced by the periodic spatial self-imaging of multimode waves, Phys. Rev. Lett., vol. 116, 183901, May 2016.

[9] L. G. Wright, Z. Liu, D. A. Nolan, M. Jun Li, D. N. Christodoulides, and F. W. Wise: Self-organized instability in graded index multimode fibre, Nat. Photon., vol. 10, pp. 771-776, Nov. 2016.

[10] S. Longhi: Modulational instability and space-time dynamics in nonlinear parabolic-index optical fibers, Opt. Lett., vol. 28, pp. 2363-2365, Dec. 2003.

[11] R. Dupiol, A. Bendahmane, K. Krupa, A. Tonello, M. Fabert, B. Kibler, T. Sylvestre, A. Barthelemy, V. Couderc, S. Wabnitz, and G. Millot: Far-detuned cascaded intermodal four-wave mixing in a multimode fiber, Optics Letters, vol. 42, pp. 1293-1296, Apr. 2017.

[12] G. Lopez-Galmiche, Z. S. Eznaveh, M. A. Eftekhar, J. A. Lopez, L. G. Wright, F. Wise, D. Christodoulides, and R. A. Correa: Visible supercontinuum generation in a graded index multimode fiber pumped at $1064 \mathrm{~nm}$, Opt. Lett., vol. 41, pp. 2553-2556, Jun. 2016.

[13] K. Krupa, C. Louot, V. Couderc, M. Fabert, R. Guenard, B. M. Shalaby, A. Tonello, D. Pagnoux, P. Leproux, A. Bendahmane, R. Dupiol, G. Millot, and S. Wabnitz: Spatiotemporal characterization of supercontinuum extending from the visible to the mid-infrared in multimode graded-index optical fiber, Opt. Lett., vol. 41, pp. 5785-5788, Dec. 2016.

[14] D. Ceoldo, K. Krupa, A. Tonello, V. Couderc, D. Modotto, U. Minoni, G. Millot, and S. Wabnitz: Second harmonic generation in multimode graded-index fibers: Spatial beam cleaning and multiple harmonic sideband generation, Optics Letters, vol. 42, pp. 971-974, Mar. 2017. 